\title{
The Powder Diffraction Mail-In Program at the Canadian Light Source: A Useful Tool for Industrial and Basic Research
}

\author{
Joel W. Reid
}

Canadian Light Source, 44 Innovation Boulevard, Saskatoon, SK, Canada

The Canadian Light Source has recently created a mail-in program for powder X-ray diffraction (PXRD) using the Canadian Macromolecular Crystallography Facility (CMCF) bending magnet beamline (08B1-1 or CMCF-BM). The program has been designed to be easy and affordable, with quick turnaround, for both industrial clients and academia. Multiple services are available ranging from basic data collection to full service options including analysis and reporting. A description of the beamline and a variety of examples including phase identification, structure solution, and quantitative phase analysis will be presented. 